\title{
"Fleeting Eclipse"-Transient Cortical Blindness (TCB) Post Coronary Angiogram: A Rare and Under-Recognised Complication
}

\section{Sugurmar ANK*, Tiau PWJ, Govindaraju S, Hassan HHC and Maskon O}

Department of Medicine, Malaysia Medical Center, Universiti Kebangsaan, Jalan Yaacob Latif, Kuala Lumpur, Malaysia

*Corresponding author: Agni Nhirmal Kumar Sugurmar, Department of Medicine, Malaysia Medical Center, Universiti Kebangsaan, Jalan Yaacob Latif, Kuala Lumpur, Malaysia, Tel: 603-9145 5555; E-mail: agninhirmal@yahoo.com

Received: June 07, 2018; Accepted: September 24, 2018; Published: September 28, 2018

Copyright: (C) 2018 Sugurmar ANK, et al. This is an open-access article distributed under the terms of the creative commons attribution license, which permits unrestricted use, distribution, and reproduction in any medium, provided the original author and source are credited.

\begin{abstract}
Transient Cortical Blindness (TCB) is a rare and alarming complication post angiographic procedures. Although the exact cause remains speculative, it is postulated that there is a direct neurotoxic effect of contrast to the occipital cortex and it is potentiated by prolonged supine position during coronary angiogram (COROS). We report a patient with cardiovascular risk factors, who underwent semi-emergency coronary intervention and suffered TCB after 22 hrs post procedure and recovered fully within $90 \mathrm{~min}$.
\end{abstract}

Keywords: Transient cortical blindness; Post coronary angiogram complications; Blind after angiography; Contrast induce encephalopathy; Post COROS blindness; TCB; Angiographic procedural related transient blindness

\section{Introduction}

Transient Cortical Blindness (TCB) has been reported during angiographic procedures-mainly cerebral angiogram, trans-lumbar aortography, myelogram and abdominal angiogram. It is uncommon to occur post coronary angiogram. This neurological adverse event is sometimes termed as contrast induced encephalopathy [1]. There are no permanent neurological sequelae in the vast majority of the cases, but it is important to recognise it as a possible complication.

\section{Case Report}

A 57-year-old gentleman was admitted to the coronary care unit with the diagnosis of unstable angina. Patient had poorly controlled hypertension and diabetes mellitus. He presented with first episode of typical chest pain, resolving spontaneously at rest.

On presentation, he was alert with blood pressure of 205/110 $\mathrm{mmHg}$, pulse rate of $100 / \mathrm{min}$, cardiovascular system examination unremarkable. A complete neurological examination was nil of note. Electrocardiogram on admission showed sinus rhythm, left axis deviation with no acute ST/T segment changes. He was admitted to the ward with Intravenous infusion of glyceryl trinitrate for blood pressure control and dual antiplatelet (oral aspirin and clopidogrel) with low molecular weight heparin (subcutaneous enoxaparin $1 \mathrm{mg} / \mathrm{kg}$ Bid) for acute coronary syndrome treatment.

In-ward stay was complicated with recurrent angina pain despite blood pressure being controlled with adequate anti-hypertensive medications. Coronary angiogram was done; showed severe single vessel disease (Figure 1). The right coronary artery was dominant with proximal to mi vessel severe stenosis of $80 \%$ to $90 \%$. A drug eluting stent (Onyx DES $3.0 \times 30 \mathrm{~mm}$ ) was placed with good final angiographic flow-TIMI flow $3.250 \mathrm{cc}$ of Iohexol $(350 \mathrm{mg}$ ), a low osmolality contrast agent was used for the procedure and this was his $1^{\text {st }}$ exposure to Iohexol. The angiographic procedure lasted for $70 \mathrm{~min}$. Patient was normotensive and asymptomatic throughout procedure.

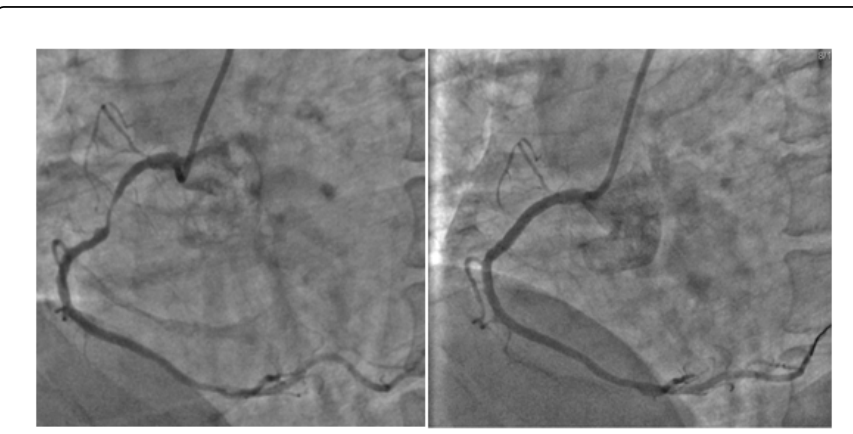

Figure 1: Coronary angiogram images. Left: Severe RCA disease, Right: Post balloon and stenting.

$22 \mathrm{hrs}$ post procedure, patient complained of blurring of vision and subsequent loss of visual acuity. Physical examination revealed an orientated patient with blood pressure of $124 / 78 \mathrm{mmHg}$, regular heart rate of 60 beats per minute. He was afebrile and had no new cutaneous manifestations such as livedo reticularis/petechiaes. Visual acuity was reduced bilaterally to just perception of light. Pupils were equal and reactive to light with no nistagmus. There was no opthalmoplegia with normal fundus examination with no relative afferent pupillary defect. There was no carotid bruiting. Full neurological examination revealed no abnormalities.

Immediate neurology unit referral was made after obtaining a noncontrasted computerized tomography (CT) of the brain for suspicion of occipital lobe/posterior circulation infarct secondary to thromboembolism (Figure 2). However, CT brain findings were normal, and symptoms lasted for 90 minutes before it spontaneously improved. Ophthalmologist review during event - commented that patient had bilateral eye moderate non-proliferative diabetic retinopathy with no papilloedema, no evidence of cherry spots to suggest central retinal artery occlusion. 
Citation: $\quad$ Sugurmar ANK, Tiau PWJ, Govindaraju S, Hassan HHC, Maskon O (2018) "Fleeting Eclipse"-Transient Cortical Blindness (TCB) Post Coronary Angiogram: A Rare and Under-Recognised Complication. J Cardiovasc Dis Diagn 6: 338. doi:10.4172/2329-9517.1000338

Page 2 of 3

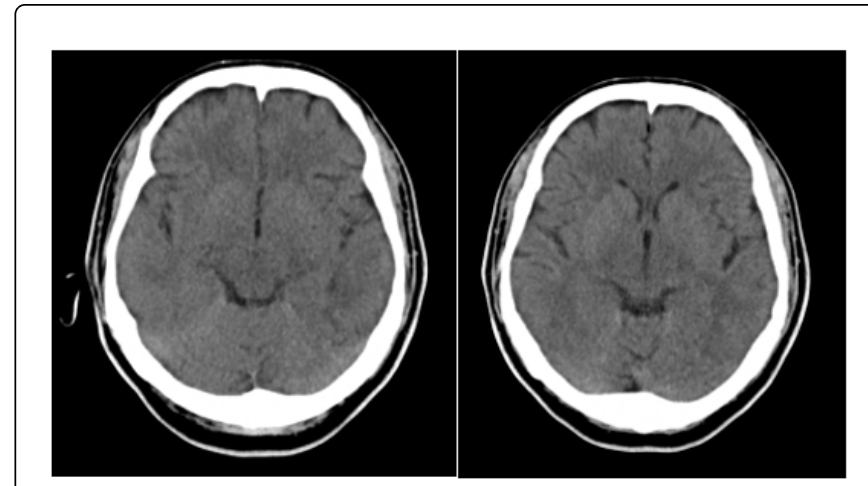

Figure 2: Plain CT of the Brain: No obvious abnormalities.

An echocardiogram showed- normal findings with no regional wall motion abnormality and no left ventricular clots. An ultrasound of both carotids was done to rule out carotid stenosis post event. An MRI brain stroke protocol was done prior to discharge-showing normal findings (Figure 3).

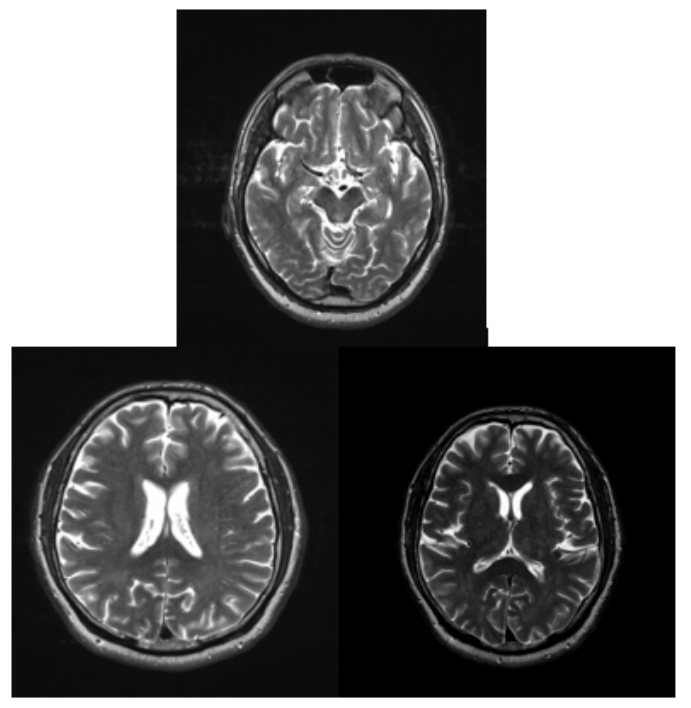

Figure 3: MRI Brain: T2 Flair, no abnormal hyperintensity signals.

The diagnosis of transient cortical blindness secondary to contrast agents was clinched. Upon discharge, there was no residual neurological deficit.

\section{Discussion}

The incidence of TCB was reported to be as high as $1 \%$ to $4 \%$ from cerebral angiography but it is less commonly seen in coronary angiogram (Estimated 0.05\%) [2]. This case report was written to highlight the rarity of this complication (Figure 4).

One of the major risk factors predisposing to this daunting complication is the type of contrast agent used. The incidence rate is much higher whenever a hyperosmolar contrast agent is used. Other notable risks include uncontrolled hypertension, hypotension, renal failure, concurrent usage of immune-suppressive drugs and dehydration [3]. History of previous coronary grafting can also be a significant risk factor for TCB [4].

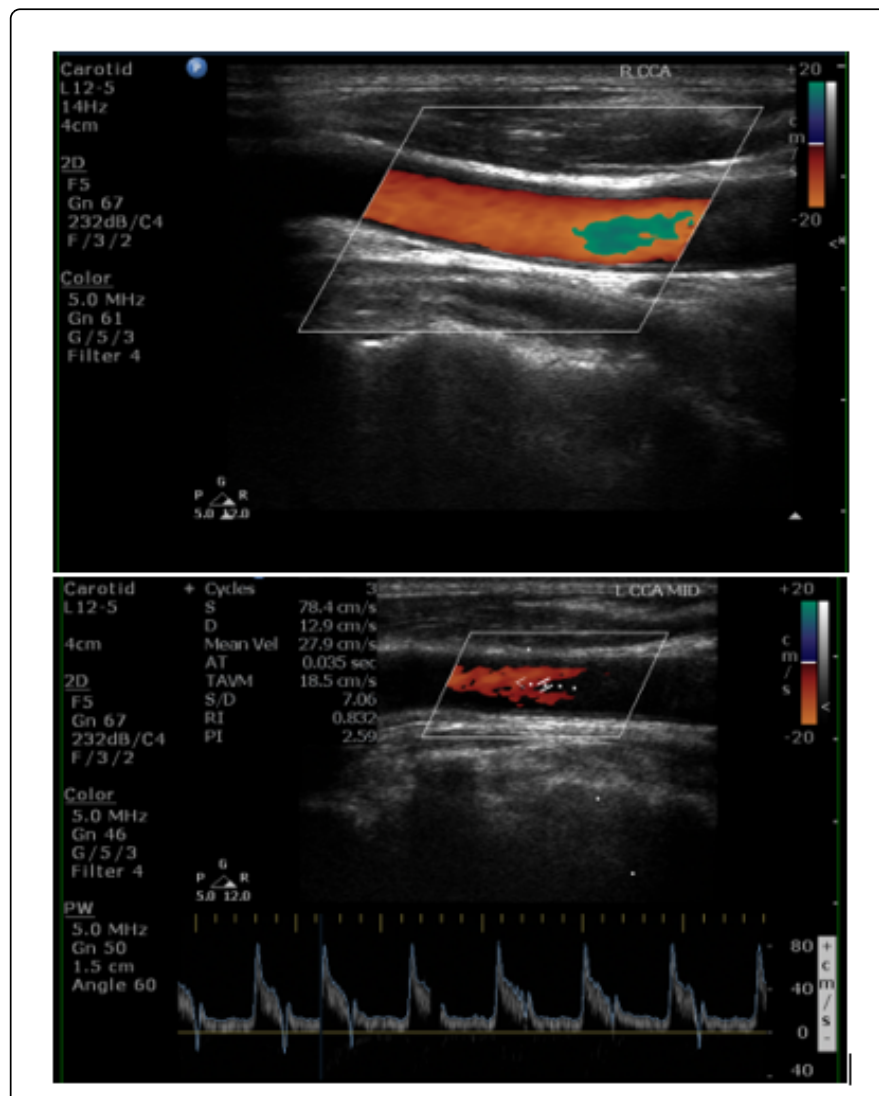

Figure 4: Ultrasound carotid Doppler, right and left carotid artery patent.

The onset of symptoms has been reported as early as a periprocedural to as late as $72 \mathrm{hrs}$ post procedure [5]. No correlation between time of symptom onset (early/late), severity of symptoms and recovery of symptoms has been documented. Symptoms subside within hours and there were reported case of full recovery taking 5-7 days. Postulated hypothesis is that TCB due to contrast resembles posterior reversible leukoencephalopathy syndrome [6]. Intra-arterial injection of contrast material crosses the Blood Brain Barrier (BBB) by endothelial pinocytosis. Posterior cerebral circulation has less sympathetic respond compared to anterior. This results in diminished vasoconstriction allowing more contrast to cross the BBB causing reversible oedema at occipital lobes [6]. Viscosity, hyperosmolarity, solubility profiles of contrast agents may play a part in the enhancement of neurotoxicity, but the role of volume is still debatable [7-9].

Brain imaging is still vital to rule out other diabolical causes of acute vision loss such as haemorrhage and embolic stroke, especially post intraarterial procedures. There are reports that even a plain CT scan may show enhancement in the occipital lobe [5]. Magnetic resonance imaging (MRI) with T2 and FLAIR would be the best modality to exclude other urgent causes. There are reported cases of FLAIR hyperintensities at the occipital region with near normal DWI (Diffusion weighted imaging) and a repeat MRI to reassess showing 
Citation: $\quad$ Sugurmar ANK, Tiau PWJ, Govindaraju S, Hassan HHC, Maskon O (2018) "Fleeting Eclipse"-Transient Cortical Blindness (TCB) Post Coronary Angiogram: A Rare and Under-Recognised Complication. J Cardiovasc Dis Diagn 6: 338. doi:10.4172/2329-9517.1000338

Page 3 of 3

complete resolution [10]. But most cases have a normal MRI finding (Figure 5). Nevertheless, MRI would still be a great tool to detect early ischemic changes especially in the event of peri-procedural transient cortical blindness. Rama et al. has reported that there is no added risk in exposing patient with previous transient cortical blindness to repeat contrast related imaging/procedures later on in life [11].

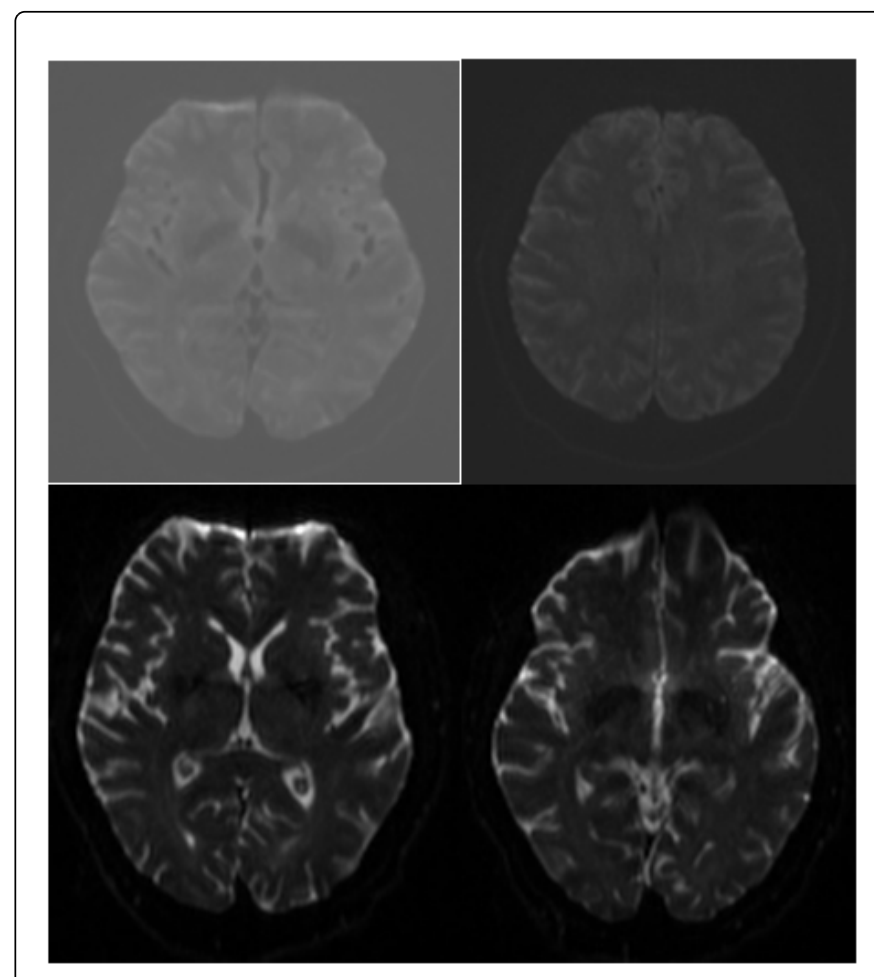

Figure 5: MRI Brain diffusion weighted and ADC sequence, unable to appreciate infarcted area especially in the occipital region.

No specific prophylactic measure has been studied. Hyper hydration was given for most patients who has suffered transient cortical blindness post procedure in the literature reviews. The relationship between hydration and attaining full recovery or speedy recovery needs to be looked into. Another aspect for further probing would be the role of corticosteroids prior to contrast introduction. Although this would be easier to gauge if the exact pathogenesis was deemed to inflammatory in nature.

\section{Conclusion}

TCB is a noteworthy complication post angiogram. Although it is reversible, modifiable risk factors should be tackled. Imaging is crucial to exclude other pathology and reassurance to patient is important.

\section{References}

1. Spina R, Simon N, Markus R, Muller DWM, Kathir K (2016) Contrast induced encephalopathy following cardiac catheterization. Catheter Cardiovasc Interv 90: 257-268.

2. Balasingam S, Azman RR, Nazri M (2016) Contrast media induced transient cortical blindness. QJM-INT J MED 109: 121-122.

3. Suri V, Agarwal R, Jadhao N, Ahuja GK (2011) Cortical blindness after contrast-enhanced CT scan in a patient of sarcoidosis: Is it related to posterior reversible encephalopathy syndrome? Ann Indian Acad Neurol 14: 298-300.

4. Yazici M, Ozhan H, Kinay O, Kilicaslan B, Karaca M, et al. (2007) Transient cortical blindness after cardiac catheterization with iobitridol. Tex Heart Inst J 34: 373.

5. Alp BN, Bozbuğa N, Tuncer MA, Yakut C (2009) Transient cortical blindness after coronary angiography. J Int Med Res 37: 1246-1251.

6. Saigal G, Bhatia R, Bhatia S, Yakut C (2004) MR findings of cortical blindness following cerebral angiography: Is this entity related to posterior reversible leukoencephalopathy? Am J Neuroradiol 25: 252-256.

7. Borghi C, Saia F, Marzocchi A, Branzi A (2008) The conundrum of transient cortical blindness following coronary angiography. J Cardiovasc Med 9: 1063-1065.

8. Niimi Y, Kupersmith MJ, Ahmad S, Song J, Berenstein A (2008) Cortical blindness, transient and otherwise, associated with detachable coil embolization of intracranial aneurysms. Am J Neuroradiol 29: 603-607.

9. Lantos G (1989) Cortical blindness due to osmotic disruption of the blood-brain barrier by angiographic contrast material CT and MRI studies. Neurology 39: 567-571.

10. Baguma M, Younan N, London F, Ossemann M, Vandermeeren Y (2017) Contrast-associated transient cortical blindness: Three cases with MRI and electrophysiology findings. Acta Neurol Belg 117: 195-199.

11. Rama BN, Pagano TV, DelCore M, Knobel KR, Lee J (1993) Cortical blindness after cardiac catheterization: Effect of rechallenge with dye. Cathet Cardiovasc Diagn 28: 149-151. 